\title{
Prévalence De Phragmanthera capitata (Spreng.) Ballé (Loranthaceae) Sur Les Hévéas Des Plantations Paysannes Du Site De L'Université Jean Lorougnon Guédé Au Centre-Ouest de la Côte d'Ivoire
}

Amon Anoh Denis-Esdras, Koulibaly Annick Victoire, Sako Hamed El Lamine, Université Jean Lorougnon Guédé, U.F.R. Agroforesterie, Daloa Côte d'Ivoire Koffi Affoué Armèle, Université Félix Houphouët Boigny, U.F.R. Biosciences Abidjan. Laboratoire de Botanique

Doi:10.19044/esj.2020.v16n21p215 URL:http://dx.doi.org/10.19044/esj.2020.v16n21p215

\section{Résumé}

En Côte d'Ivoire, l'hévéaculture est en pleine extension à cause de ses retombées économiques et financières. Malheureusement, une destruction actuelle des branches de nombreux individus d'hévéas par les Loranthaceae, notamment Phragmanthera capitata est constatée. Cette situation est préjudiciable à l'économie de cette activité. Pour limiter l'infestation de ces parasites, il est urgent d'entreprendre des méthodes de lutte efficaces. Pour mieux contrôler les Loranthaceae, une meilleure connaissance de leur comportement s'impose. Cette étude vise à évaluer la prévalence de $P$. capitata sur les hévéas des plantations paysannes du site de l'Université Jean Lorougnon Guédé de Daloa. Les inventaires basés sur les méthodes de relevés de surface et des observations directes ont été réalisés dans 3 plantations d'hévéas. Les résultats ont montré une incidence élevée et une sévérité d'infestation des hévéas par $P$. capitata. Ils ont indiqué une différence significative entre l'incidence d'infestation et l'indice de sévérité des hévéas en fonction du dbh. L'incidence d'infestation de ce parasite sur les hévéas des plantations paysannes du site de l'Université est de 30,48 $\pm 11,12 \%$ et l'indice de sévérité est $34,16 \pm 15,30 \%$. Ces résultats pourraient servir de référence pour la recherche d'une méthode de lutte appropriée contre ce parasite très envahissant et très dévastateur sur les hévéas. 
Keywords: Hévéa, Phragmanthera capitata, Incidence, Sévérité d'infestation, Côte d'Ivoire, Afrique de l'Ouest

\title{
Prevalence of Phragmanthera Capitata (Spreng.) Ballé (Loranthaceae) on Rubber Trees of Farming Plantations at the Jean Lorougnon Guédé University Site in Central West of Côte d'Ivoire
}

Amon Anoh Denis-Esdras, Koulibaly Annick Victoire, Sako Hamed El Lamine, Université Jean Lorougnon Guédé, U.F.R. Agroforesterie, Daloa Côte d'Ivoire

Koffi Affoué Armèle, Université Félix Houphouët Boigny, U.F.R. Biosciences Abidjan. Laboratoire de Botanique

\begin{abstract}
In Cote d'Ivoire, rubber cultivation is booming due to its economic and financial benefits. Unfortunately, the destruction of individual rubber tree branches notably by Loranthaceae, Phragmanthera capitata has been noticed as an impediment to rubber cultivation. This situation is detrimental to the economic rentability of this activity. Developing effective strategies to control these parasites plants is urgent. A better understanding of the behavior of Loranthaceae is necessary to control their expansion. This study aims to evaluate the prevalence of $P$. capitata on the rubber trees of farming plantations located at the Jean Lorougnon Guédé University site in Daloa. The inventories based on sampling blocks method and direct observations were used in three rubber plantations. The results showed a high level and severity of $P$. capitata invasion on rubber trees and indicated a significant difference between the incidence and intensity on rubber trees depending on their dbh. The incidence of invasion of $P$. capitata on rubber trees of the studied farming plantations premises is of $30,48 \pm 11.12 \%$ and the severity index is $34,16 \pm 15.30 \%$. These results could be used as a reference to find more appropriate solutions to fight against the invasive and devastating effects of this parasite on rubber trees.
\end{abstract}


Keywords: Rubber tree, Phragmanthera capitata, Incidence, Severity of infestation, Côte d'Ivoire, West Africa

\section{Introduction}

L'hévéa: Hevea brasiliensis (Künth) Mull. Arg. de la famille des Euphorbiaceae est un arbre cultivé dans le monde tropical pour son latex. En Côte d'Ivoire, l'hévéaculture, depuis les années 1990 joue un rôle majeur dans le tissu socio-économique du pays (Tadjau, 2008). L'intérêt porté à cette culture repose essentiellement sur ses retombées économiques importantes pour les hévéaculteurs et pour le pays. En effet, en 2008, l'exportation annuelle ivoirienne du caoutchouc naturel s'élevait à 256000 tonnes pour une valeur de 41,7 milliards de F CFA (BNETD, 2008). La Côte d'Ivoire est le premier producteur africain du caoutchouc naturel et le 7ème au plan mondial (Symenouh, 2008).

Malheureusement, l'hévéaculture est soumise d'année en année à plusieurs contraintes parasitaires qui affectent souvent la croissance optimale des plants et surtout à la durabilité des exploitations hévéicoles. Au nombre de ces contraintes parasitaires se trouvent les attaques de plus en plus marquées des plantes épiphytes hémiparasites de la famille des Loranthaceae qui causent des dommages considérables aux espèces cultivées et spontanées dans le monde en général et, en particulier en Afrique subsaharienne (Gill \& Onybe, 1990; Hévécam, 1995). Aujourd'hui, en sillonnant les campagnes ivoiriennes, ces végétaux parasites qui envahissent en grand nombre les pieds d'hévéas, sont perceptibles par la couleur et la forme en buchette d'allumette de leurs fleurs (Traoré et al., 2003). Dans le monde, la famille des Loranthaceae comprend 950 espèces réparties dans 77 genres (Polhill \& Wiens, 1998). En Afrique, la taxonomie révèle l'existence de 230 espèces appartenant à 21 genres dont 7 sont identifiés en Afrique de l'Ouest (Engone Obiang \& Sallé, 2006). En Côte d'Ivoire, on note 24 espèces dont 19 sont signalées dans le Sud forestier (Aké-Assi, 2001). Plusieurs travaux réalisés en Afrique sur les Loranthaceae ont signalé une grave infestation des hévéas par Phragmanthera capitata (Spreng.) Ballé (Loranthaceae) connue pour être un ravageur dans les exploitations hévéicoles (Gill \& Onybe, 1990; Hévécam, 1995; Koffi, 2004; Engone Obiang \& Salle, 2006; Soro et al., 2010; Amon, 2014). Or, à l'état actuel des connaissances, la prévalence et le degré d'infestation de P. capitata sur les hévéas reste peu d'étudiée et les informations sur le degré de son infestation dans les exploitations hévéicoles dans le Centre-Ouest de la Côte d'Ivoire sont rares. Cette étude vise donc à déterminer l'incidence et la sévérité d'infestation de P. capitata sur les hévéas dans les plantations paysannes du site de l'Université Jean Lorougnon Guédé. 


\section{Matériel et Méthodes}

\section{Milieu d'étude}

L'étude a été conduite dans les plantations paysannes d'hévéas du site de l'Université Jean Lorougnon Guédé dans la ville de Daloa (figure 1), au Centre-Ouest de la Côte d'Ivoire de coordonnées géographiques $6^{\circ} 27^{\prime} 00^{\prime \prime}$ de latitude Nord et 5 56'00" de longitude Ouest. La ville de Daloa est située à 141 $\mathrm{km}$ de Yamoussoukro, la capitale politique de la Côte d'Ivoire. Elle abrite l'Université Jean Lorougnon Guédé, notre zone d'étude. La zone d'étude se situe dans le domaine Guinéen caractérisé par un régime climatique de type équatorial comprenant deux saisons de pluies et deux saisons sèches (Eldin, 1971). Les précipitations mensuelles varient entre $1300 \mathrm{~mm}$ et $1800 \mathrm{~mm}$. La région de Daloa appartient au secteur mésophile dont la végétation est composée de forêts denses humides semi-décidues, de forêts défrichées et de savanes mésophiles (Guillaumet \& Adjanohoun, 1971), ces formations végétales abritant par ailleurs, de nombreuses espèces animales (antilopes, hippopotames) et espèces ligneuses. La forte pression démographique a conduit à la dégradation progressive des forêts au profit des plantations agricoles telles que les cacaoyers, les caféiers, les hévéas, les anacardiers, etc. (Koulibaly, 2019).

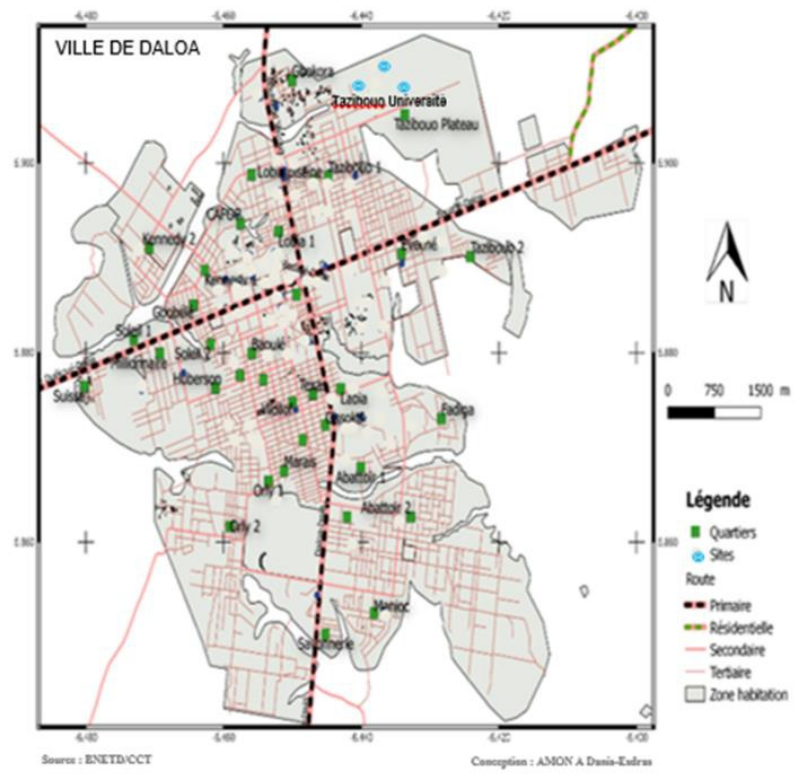

Figure 1 : Localisation de la zone d'étude dans la ville de Daloa

\section{Matériel et Méthodes}

\section{Matériel}

Le matériel biologique était composé de P. capitata et des pieds d'hévéas. Le matériel technique comprend un appareil de positionnement 
géographique (GPS), un appareil photo numérique, un ruban-mètre, du fil de sisal, des piquets et des fiches de relevés.

\section{Méthode}

\section{Collecte de données}

Pour la présente étude, la méthode de relevés de surface qui consiste à recenser les pieds des hévéas rencontrés sur des superficies carrées ou rectangulaires (Hall \& Swaine, 1981) couplée à des observations directes en vue de noter la présence ou l'absence du parasite (Soro, 2010) ont été adoptées dans trois plantations d'hévéas: plantation $1\left(06^{\circ} 55^{\prime} 41.7^{\prime \prime} \mathrm{N}\right.$ et $\left.006^{\circ} 25^{\prime} 25.6^{\prime \prime} \mathrm{W}\right)$, plantation $2\left(06^{\circ} 51^{\prime} 39.5^{\prime \prime} \mathrm{N}\right.$ et $\left.006^{\circ} 22^{\prime} 33.15^{\prime \prime} \mathrm{W}\right)$ et plantation $3\left(06^{\circ} 41^{\prime} .18^{\prime} \mathrm{N}\right.$ et $\left.006^{\circ} 26^{\prime} .13^{\prime} \mathrm{W}\right)$. L'inventaire a consisté à dénombrer d'héveas non parasités et ceux parasités, le parasite et le nombre de ses touffes sur chaque pied d'hévéa a été réalisé à partir de deux parcelles de relevé de $60 \mathrm{~m}$ $\times 40 \mathrm{~m}\left(2400 \mathrm{~m}^{2}\right)$ délimitées dans chaque plantation (figure 2).

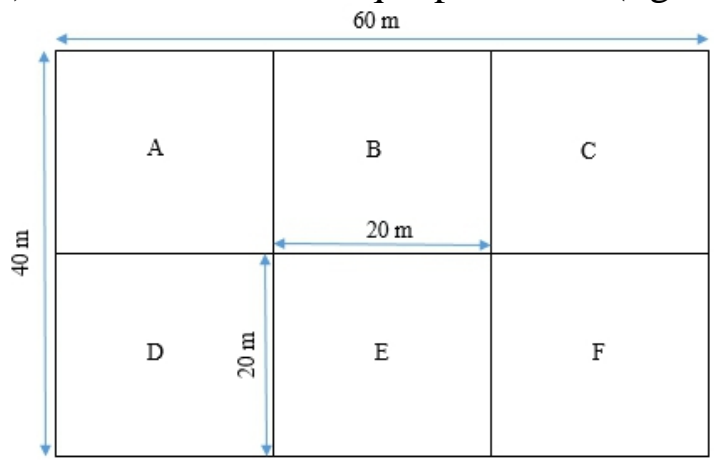

Figure 2: Schéma du dispositif de relevés

Pour faciliter l'inventaire des pieds d'hévéas et le dénombrement des touffes du parasite sur les individus infestés, la placette unitaire de $2400 \mathrm{~m}^{2}$ a été subdivisée en 6 sous-parcelles carrées (A, B, C, D et E) de 20 m x 20 m (400 $\mathrm{m}^{2}$ ) chacune (figure 2 ).

La hauteur totale des pieds d'hévéas et de leur diamètre à hauteur de poitrine (dbh à $1,30 \mathrm{~m} \mathrm{du}$ sol) ont été également relevés. La présence ou l'absence du parasite et le nombre de ses touffes par pied d'hévéa ont été relevés. Les données recueillies ont permis de déterminer l'incidence d'infestation de $P$. capitata dans les trois plantations selon la formule utilisée par Asare-Bediako et al. (2013):

$$
\text { Incidence d'infestation }(\%)=\frac{\text { Nombre de plants infestés de Loranthaceae }}{\text { Nombre total de plants évalués dans la plantation }} \times 100
$$


Pour évaluer la sévérité de l'infestation de $P$. capitata sur les plantations d'hévéas, 10 pieds ont été sélectionnés de manière aléatoire dans une sousparcelle (E) de $400 \mathrm{~m}^{2}$, soit 20 pieds par plantation. Au total, 60 pieds d'hévéas ont été sélectionnés au hasard dans les trois plantations. La sévérité de l'infestation sur chaque pied d'hévéa a été évaluée à partir d'un indice de gravité d'infestation de 0 à 4 en fonction du nombre de touffes de ce parasite (tableau 1).

Tableau 1 : Echelle d'infestation du parasite

\begin{tabular}{|c|c|c|}
\hline Indice de gravité d'infestation & Note & Nombre de touffes/pied d'hévéa \\
\hline Aucune infestation & 0 & Pas de touffes de Loranthaceae \\
\hline Infestation légère & 1 & $1-3$ touffes par pied \\
\hline Infestation modérée & 2 & $4-6$ touffes par pied \\
\hline Infestation sévère & 3 & $7-9$ touffes par pied \\
\hline Très grave & 4 & Nombre de touffes $>9$ par pied \\
\hline
\end{tabular}

Les indices de sévérité $(I S)$ pour chaque plantation ont été calculés à l'aide de la formule suivante (Mohamed et al., 2013):

$$
I S=\frac{(0 \times a)+(1 \times b)+(2 \times c)+(3 \times d)+(4 \times e)}{a+b+c+d+e} \times \frac{100}{4}
$$

où $a, b, c, d$ et $e$ sont le nombre de pieds d'hévéas entrant dans les catégories d'infections 0 - 4 établis.

\section{Analyses statistiques}

Les données générées ont été soumises à l'analyse de variance (ANOVA) à une voie pour comparer les valeurs moyennes d'incidence et sévérité d'infestation de $P$. capitata sur les hévéas. L'analyse statistique a été faite à l'aide du logiciel STATISTICA version 7.1. Ce programme prévoit, en cas de différences significatives, une comparaison des moyennes (Dagnelie, 1980), par le test de Duncan au seuil de 5\% $(\alpha<0,05)$.

\section{Résultats}

\section{Incidence et sévérité d'infestation de $\boldsymbol{P}$. capitata sur les hévéas.}

Les valeurs de l'incidence d'infestation des hévéas par $P$. capitata enregistrée dans les plantations paysannes du site de l'Université Jean

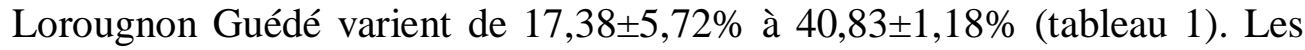
hévéas de la plantation 3 (P13) sont les plus parasités avec une incidence d'infestation de l'ordre de 40,83 $\pm 1,18 \%$. Viennent ensuite ceux de la

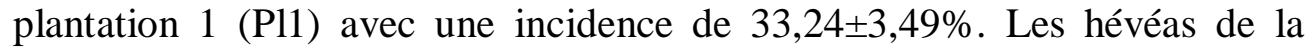
plantation 2 (P12) sont les moins infestés $(17,38 \pm 5,72 \%)$. 
Tableau 1 : Incidence d'infestation de $P$. capitata sur les hévéas dans les plantations

\begin{tabular}{|l|l|l|l|l|}
\hline $\begin{array}{l}\text { Plantations } \\
\text { (Hévéas) }\end{array}$ & $\begin{array}{l}\text { Nombre total } \\
\text { pieds/parcelle }\end{array}$ & $\begin{array}{l}\text { Nombre pieds } \\
\text { parasités/parcelle }\end{array}$ & $\begin{array}{l}\text { Incidence } \\
(\%) / \text { parcelle }\end{array}$ & $\begin{array}{l}\text { Incidence } \\
(\%) / \text { plantation }\end{array}$ \\
\hline \multirow{2}{*}{ Pl1 } & 14 & 5 & 35,71 & \multirow{2}{*}{$33,24 \pm 3,49$} \\
\cline { 2 - 4 } & 13 & 4 & 30,77 & \multirow{2}{*}{$17,38 \pm 5,72$} \\
\hline \multirow{2}{*}{ P12 } & 15 & 2 & 13,33 & \multirow{2}{*}{$40,83 \pm 1,18$} \\
\cline { 2 - 5 } & 14 & 3 & 21,43 & $30,48 \pm 11,12$ \\
\cline { 2 - 5 } Pl3 & 12 & 5 & 41,67 & \\
\hline Total & 15 & 6 & 40 & \\
\hline
\end{tabular}

L'analyse de variance effectuée indique effectivement deux groupes statistiques différents (figure 3). Le groupe 1, le plus parasité est constitué des hévéas de Pl1 et Pl3. Le groupe 2, le moins parasité est composé des hévéas de $\mathrm{P} 12(\mathrm{p}=0,02)$.

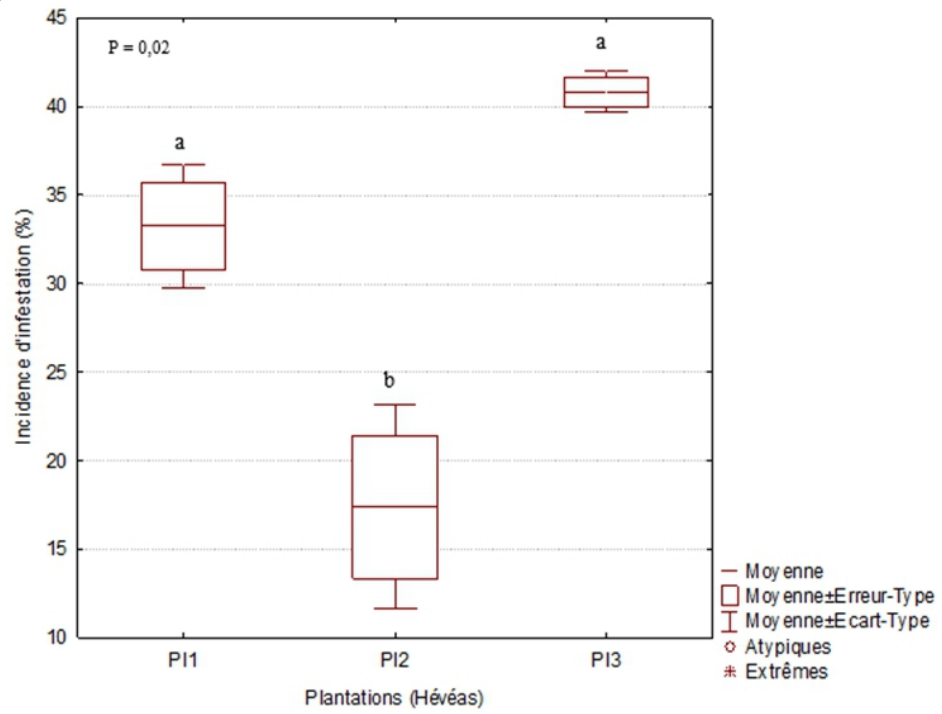

Figure 3 : Incidence d'infestation des hévéas par P. Capitata

L'incidence d'infestation de $P$. capitata sur les hévéas des plantations paysannes du site de l'Université Jean Lorougnon Guédé pris ensemble est de l'ordre de $30,48 \pm 11,12 \%$.

Les indices de sévérité d'infestation de $P$. capitata sur les hévéas évalués, oscillent entre $17,50 \pm 10,60 \%$ et $47,50 \pm 3,53 \%$ (tableau 2). Les hévéas de la plantation 3 sont les plus infestés, avec un indice de sévérité moyenne d'infestation de $47,50 \pm 3,53 \%$. Viennent ensuite ceux de la plantation 1 $(37,50 \pm 10,60 \%)$. 
Tableau 2 : Indice de sévérité d'infestation de P. capitata sur les hévéas dans les plantations

\begin{tabular}{|l|l|l|}
\hline \multirow{2}{*}{ Plantations (Hévéas) } & Indice de sévérité /parcelle & $\begin{array}{l}\text { Indice } \\
\text { sévérité } \\
(\%) / p l a n t a t i o n\end{array}$ \\
\hline \multirow{2}{*}{ P11 } & 30 & \multirow{2}{*}{$37,50 \pm 10,60$} \\
\cline { 2 - 3 } & 45 & $17,50 \pm 10,60$ \\
\hline \multirow{2}{*}{ P12 } & 10 & $47,50 \pm 3,53$ \\
\hline \multirow{2}{*}{$\mathrm{Pl} 3$} & 25 & $34,16 \pm 15,30$ \\
\cline { 2 - 3 } & 50 & \\
\hline
\end{tabular}

L'indice de sévérité d'infestation des hévéas de la plantation 2 est moins important (tableau 2). L'analyse de variance effectuée indique 3 groupes statistiquement différents (figure 4). La sévérité d'infestation de $P$. capitata sur les hévéas des plantations paysannes du site de l'Université Jean Lorougnon Guédé prise ensemble est de 34,16 $\pm 15,30 \%$.

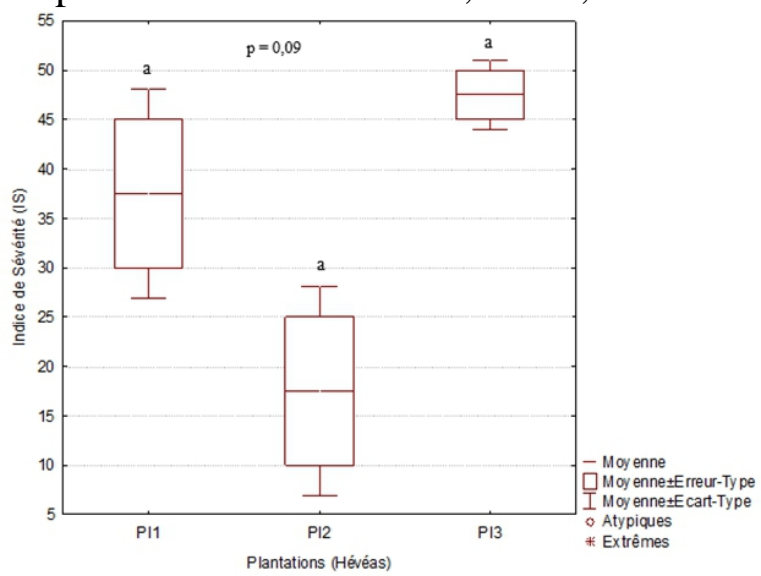

Figure 4 : Sévérité d'infestation de $P$. capitata sur les hévéas dans les plantations

\section{Incidence et sévérité d'infestation en fonction des diamètres et des hauteurs}

L'incidence d'infestation de P. capitata en fonction des classes de diamètres des hévéas parasités des plantations paysannes du site de l'Université Jean Lorougnon Guédé, évaluée montre que ce parasite attaque le plus d'individus à diamètre moyen et gros avec des valeurs d'incidences élevées. L'analyse de variance de la structure horizontale réalisée indique effectivement une différence significative $(\mathrm{p}=0,03)$ entre 3 groupes statistiquement différents $\mathrm{a}$, $\mathrm{ab}$ et $\mathrm{b}$ (figure 5 ). L'incidence des classes de diamètres (16-20) et (>20) se distingue nettement des trois autres classes statistiquement. S'agissant de l'analyse de la structure verticale, elle a montré aussi une différence significative entre l'incidence d'infestation du parasite sur les hévéas en fonction de leurs classes de hauteurs $(p=0,07)$. Les incidences 
d'infestation se distinguent statistiquement à travers 3 groupes homogènes a, $\mathrm{b}$ et $\mathrm{c}$ pour les différentes classes de hauteurs des hévéas. L'incidence d'infestation de la classe (>20) est statistiquement plus élevée (figure 6).

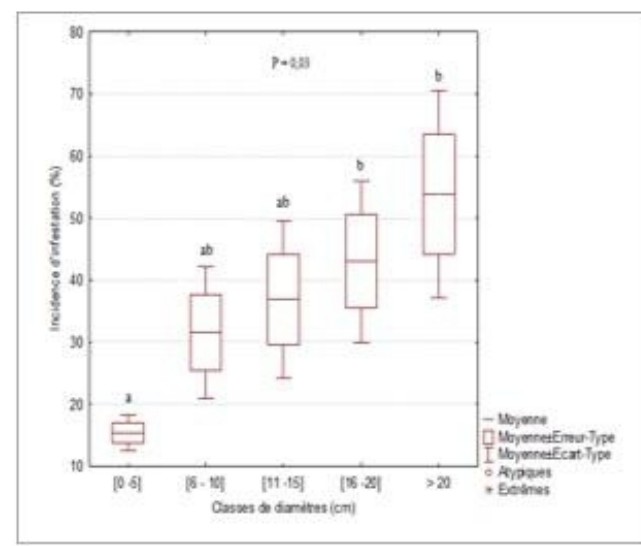

Figure 5 : Incidence en fonction des classes de diamètres des hévéas

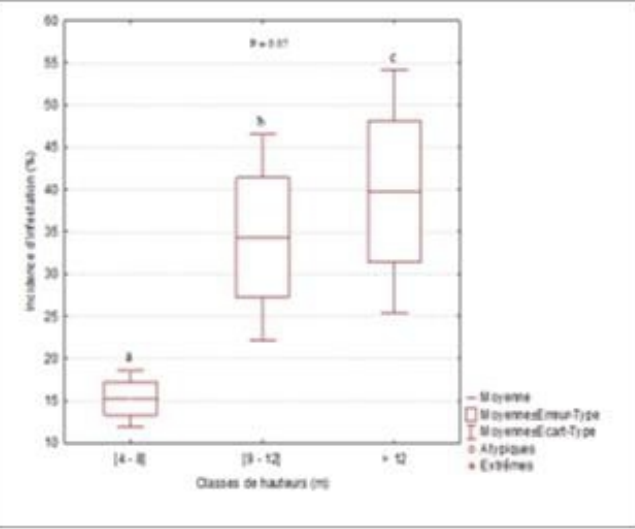

Figure 6 : Incidence en fonction des classes de hauteurs des hévéas

La sévérité d'infestation des hévéas par $P$. capitata évaluée est numériquement élevée en fonction des classes de diamètres. L'analyse de la structure horizontale montre effectivement une différence significative entre 5 groupes statistiquement différents $a, a b, a b c, b c$ et $c$ entre les indices de sévérité d'infestation des hévéas (Figure 7). Toutes les classes de diamètres se distinguent statiquement entre elles au niveau du groupe statistique $(p=0,01)$. Aussi, l'analyse de variance de la structure verticale révèle-t-elle une différence significative $a, a b$ et $b(p=0,07)$ entre les valeurs de sévérité d'infestation des hévéas affectés (Figure 8).

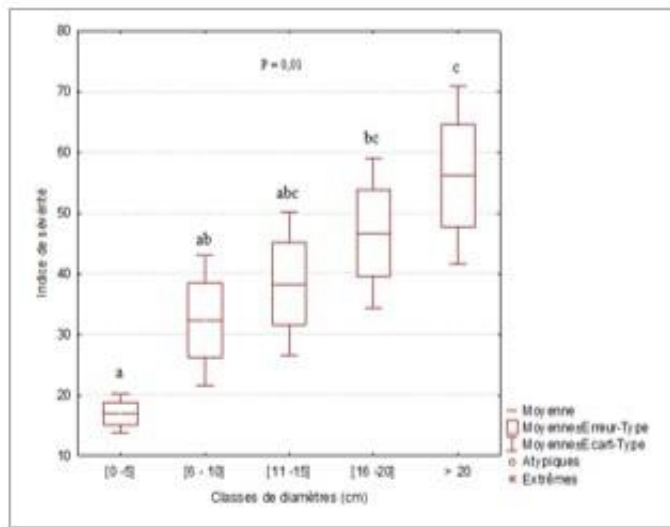

Figure 7 : Sévérité d'infestation en fonction des classes de diamètres des hévéas

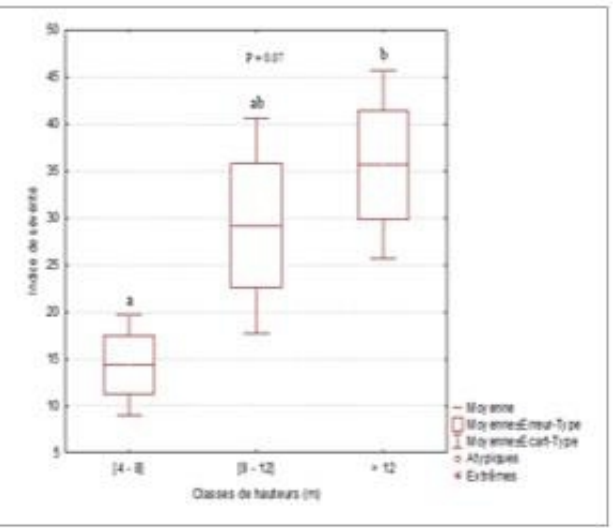

Figure 8 : Sévérité d'infestation en fonction des classes de hauteurs des hévéas 


\section{Discussion}

L'incidence d'infestation de Phragmanthera capitata sur les hévéas des plantations paysannes du site de l'Université Jean Lorougnon Guédé a été évaluée. Les trois plantations échantillonnées prises ensemble ont enregistré des pourcentages d'incidences d'infestation compris entre 33,24 $\pm 3,49$ et $40,83 \pm 1,18 \%$. Ces pourcentages sont nettement supérieurs à celui de 10,40\% observé par Koffi (2004) sur des hévéas à Anguédedou au Sud de la Côte d'Ivoire et celui de 14,10\% rapporté par Soro et al. (2010) dans les plantations d'hévéas des sous-préfectures de Gagnoa et d'Ouragahio en zone forestière du pays. Mais ce pourcentage d'incidence est inférieur à celui de Mohamed et al. (2013) qui oscillait entre 23 et $73 \%$ dans les plantations de goyaves de Gezira State, au Sudan et celui de Asare-Bediako et al. (2013) qui se situe entre 84 et $100 \%$ dans les plantations d'oranges à Abura-Asebu-Kwamankese, au Centre du Ghana. Cette différence relevée pourrait s'expliquer par la différence des zones explorées dont certaines seraient plus favorables à l'infestation aux Loranthaceae, mais aussi aux zones climatiques.

Les indices de sévérité d'infestation des plantations d'hévéas par ce

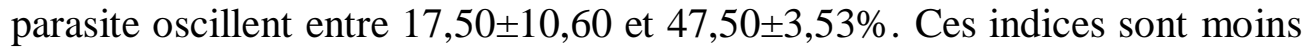
élevés que ceux de Asare-Bediako et al. (2013) compris entre $20 \%$ et $90 \%$ obtenus dans les plantations d'oranges au Ghana. Ceci serait lié au de type de culture et à l'âge des plantations.

L'incidence et l'indice de sévérité d'infestation évalués en fonction des classes de diamètres et de hauteurs des pieds d'hévéas parasités montrent que $P$. capitata attaque plus les individus à gros diamètre présentant une grande hauteur. Ces résultats confirment ceux de Soro et al. (2010) et de Ondoua et al. (2015).

La valeur d'incidence $P$. capitata enregistrée sur les hévéas est l'ordre de $30,48 \pm 11,12 \%$ et la sévérité d'infestation est de $34,16 \pm 15,30 \%$ dans les plantations paysannes du site de l'Université Jean Lorougnon Guédé. Ces résultats confirment les travaux de Gill \& Onybe (1990), Hévécam (1995), Koffi (200), Engone et al. (2006) et Soro et al. (2010) qui ont déjà signalé l'aspect très envahissant et très dévastateur de ce parasite sur les individus d'hévéas en Afrique. Aussi, ces résultats préconisent-ils en amont une lutte énergique contre ce parasite nuisible sur les pieds d'hévéas parasités en Afrique.

\section{Conclusion}

Les résultats obtenus montrent que l'espèce de Loranthaceae Phragmanthera capitata est infeste densément les hévéas des plantations paysannes du site de l'Université Jean Lorougnon Guédé. L'incidence et la sévérité d'infestation du parasite sur les hévéas sont élevées. Les valeurs d'incidence et de sévérité d'infestation du parasite sur les hévéas sont 
respectivement de $30,48 \pm 11,12 \%$ et $34,16 \pm 15,30 \%$. La prévalence $P$. capitata sur les hévéas est fonction du diamètre et de la hauteur des individus parasités. Ces résultats pourraient servir de données pour mettre en place un programme de recherche de lutte ciblée. Aussi, suggèrent-ils des recherches de clones résistants aux Loranthaceae.

\section{References:}

1. Aké-Assi L., 2001. Flore de la Côte d'Ivoire 1, catalogue, systématique, biogéographie et écologie. Conservatoire et Jardin Botanique, Genève, Switzerland, Boissiera 57, $396 \mathrm{p}$.

2. Amon ADE, 2014. Les Loranthaceae (guis), hémiparasites vasculaires des arbres et des arbustes des agroécosystèmes de la région du SudComoé, en zone de forêt dense sempervirente de la Côte d'Ivoire. Mémoire de Thèse de l'Université Félix Houphouët-Boigny, option Agroforesterie, $213 \mathrm{p}$.

3. Asare-Bediako E., Addo-Quaye AA., Tetteh JP., Buah JN., Van Der Puije GC., Acheampong RA., 2013. Prevalence Of Mistletoe On Citrus Trees In The Abura-Asebu-Kwamankese District Of The Central Region Of Ghana. International Journal of Scientific \& Technology Research (2) 7: 122-127.

4. BNETD, 2008. Présentation de la Côte d'Ivoire. http://www.bnetd.ci/côte d'ivoire agriculture.htm. [Consulté le $07 / 08 / 2009$ ].

5. Dagnelie P., 1980. Théorie et méthodes statistiques, application agronomique. Vol. 2. Gembloux, Belgique: Presses agronomiques de Gembloux. http://www.dagnelie.be/autrepub.html. [Consulté le 25/02/2020].

6. Eldin M., 1971. Le climat in : Avenard JM., Girard G., Siroulon J., Ouchebeuf P., Guillaumet JL., Adjanohoun E., Perraud A. (eds). Le Milieu naturel de Côte d'Ivoire, O.R.S.T.OM., Paris: 77-108.

7. Engone Obiang NL. \& Sallé G., 2006. Faut-il éradiquer Phragmanthera capitata, parasite des hévéas en Afrique ? Laboratoire de parasitologie végétale, Université Pierre et Marie-Curie, Paris (France). Compte Rendus (C. R.) Biologies, 329 : 185-195. http://france.elsevier.com/direct/CRASS3.

8. Gill LS. \& Onybe HI., 1990. Mistletoes on rubber trees in Nigeria. Haustorium, 23: 1-2. Guyot J. \& Ntawanga Omanda E., 1998. Note sur la sensibilité de six classes d'hévéa à Phragmanthera capitata. Plantation, Recherche, Développement (5)5 : 356-361. Hévécam (Hévéa du Cameroun) : 1995. Rapport de la direction exploitation agricole : bilan de l'essai Loranthus 12-AGRO/DEA, 9 p. 
9. Guillaumet, JL. \& Adjanohoun E., 1971. La Végétation de la Côte d'Ivoire. In: Le Milieu naturel de Côte d'Ivoire. Édition O.R.S.T.OM. Paris : $157-266$.

10. Hall JB. \& Swaine MD., 1981. Distribution and ecology of vascular plants in a rain forest vegetation in Ghana. Geobotany, $383 \mathrm{p}$.

11. Koffi AA., 2004. Évaluation de l'incidence des Loranthaceae sur la productivité de Hevea brasiliensis (Kunth) Müll. Arg. à Anguédédou (Sud de la Côte d'Ivoire). Mémoire de D.E.A. de Botanique, Université de Cocody-Abidjan, $52 \mathrm{p}$.

12. Koulibaly A., 2019. Développement agricole durable: La phytodiversite comme outil de gestion des plantations de cultures de rente en Côte d'Ivoire. Agronomie Africaine, $11 \mathrm{p}$.

13. Mohamed S., Zaroug, Abbasher, A., Abbasher and Eldur B., Zahran, 2013. Incidence and severity of the mistletoe Tapinanthus globiferus on guava and lime and its mechanical control in the Gezira State, Sudan. Journal of Science and Technology (14) : 44-51.

14. Ondoua Joseph Marie1, Dibong Siegfried Didier, Taffouo Victor Désiré \& Ngotta Biyon Jacques Bruno, 2015. Parasitisme des champs semenciers de cacaoyers par les Loranthaceae dans la localité de Nkoemvone (sud Cameroun). Journal of Applied Biosciences, 85: 7794-7803.

15. Polhill R. \& Wiens D., 1998. Mistletoes of Africa, The Royal Botanic, Kew: $370 \mathrm{p}$.

16. Salle G., 2004. Les plantes parasites, 14 p. http://www. futurasciences.com/magazines/botanique plantes parasites. [Consulté le $17 / 01 / 2020]$.

17. Soro D., 2006. Variabilité de quelques caractères morphologiques, production en fruits et moyen de lutte, par émondage, contre les Loranthaceae (Plantes vasculaires parasites) du karité : cas du parc naturel à karités de Tengrela, dans le Nord de la Côte d'Ivoire. Mémoire de Thèse de l'Université de Cocody-Abidjan, option Agroforestérie: $136 \mathrm{p}$.

18. Soro K., Soro D., N’Guessan K., Gnahoua GM. \& Traoré D., 2010. Parasitisme des Loranthaceae sur les hévéas en zone forestière des sous-préfectures de Gagnoa et d'Ouragahio, en Côte d'Ivoire. Journal of Animal et Plant Sciences: 597- 604.

19. Soro K., 2010. Les Loranthaceae (guis) des agroécosystèmes dans l'Ouest de la Côte d'Ivoire: flore, parasitisme et usages dans les Départements de Oumé, de Gagnoa et de Soubré. Mémoire de Thèse de l’Université de Cocody-Abidjan, option Agroforestérie, 183p. 
20. Symenouh V., 2008. Hévéa: Une culture d'exportation en pleine croissance. Mission économique de l'ambassade de France en Côte d'Ivoire, Ubifrance et les missions économiques, $3 \mathrm{p}$.

21. Tadjau P., 2008. Actualité. Le repère du mars 2008. Abidjan.net Côte d'Ivoire. Matières premières: L'hévéa, le nouveau Cacao ivoirien. www.monhevea.com/l-hevea-le-nouveaucacao-ivoirien.html [Consulté le 19/02/2020].

22. Traoré D., Da KP. \& Soro D., 2003. Lutte contre les plantes vasculaires parasites du Karité, dans le Nord de la Côte d'Ivoire. Cas du parc naturel à karités de Tengrela. Université de Cocody. U.F.R. Biosciences. Laboratoire de Botanique. Rapport du P.E.P., A.I.S.A.CI, $116 \mathrm{p}$. 